\title{
Lipoprotein (a) and Venous Thromboembolism in Adults: A Meta-Analysis
}

\author{
Francesco Sofi, MD, PhD, ${ }^{a}$ Rossella Marcucci, MD, PhD, ${ }^{a}$ Rosanna Abbate, MD, ${ }^{a}$ Gian Franco Gensini, MD, ${ }^{a, b}$ \\ Domenico Prisco, MDa \\ ${ }^{a}$ Department of Medical and Surgical Critical Care, Thrombosis Centre; Centre for the study at molecular and clinical level of \\ chronic, degenerative and neoplastic diseases to DEvelop NOvel THErapies, University of Florence, Italy; ${ }^{b}$ Don Carlo Gnocchi \\ Foundation, Centro S. Maria agli Ulivi, Onlus IRCCS, Florence; Azienda Ospedaliero-Universitaria Careggi, Florence, Italy.
}

\begin{abstract}
BACKGROUND: $\quad$ Lipoprotein (a) [Lp(a)], a low-density lipoprotein particle linked to apolipoprotein (a), has been recently demonstrated to be an independent risk factor for arterial vascular diseases. However, despite increasing evidence of the association between high $\mathrm{Lp}(\mathrm{a})$ and arterial thrombotic diseases, few and conflicting results on the association between high $\mathrm{Lp}$ (a) levels and venous thromboembolism have been obtained. The aim of this article is to systematically examine the published data on the association between high Lp(a) levels and venous thromboembolism.

METHODS: A systematic search of all publications listed in the electronic databases (Medline, EMBASE, Web of Science, and The Cochrane Library) up to November 2006, using keywords in combination both as MeSH terms and text words, was conducted.

RESULTS: Six case-control studies were included, incorporating 1826 cases of venous thromboembolism and 1074 controls. The summary odds ratios of included case-control studies under a fixed-effects model showed a statistically significant association between $\mathrm{Lp}$ (a) levels $>300 \mathrm{mg} / \mathrm{L}$ and venous thromboembolism: $1.87,95 \%$ confidence interval (CI), 1.51-2.30; $P<.0001$. Furthermore, a random-effects model, which accounts for the interstudy variation, yielded a similar estimate of increased risk (odds ratio [OR] 1.77; 95\% CI, 1.14-2.75; $P=.01$ ).

CONCLUSIONS: The present meta-analysis shows a significant association between high Lp(a) levels and the occurrence of venous thromboembolism in adults. Indeed, the detection of Lp(a) could be of clinical relevance for venous thromboembolism, especially among patients with absence of traditional and thrombophilic risk factors. (c) 2007 Elsevier Inc. All rights reserved.
\end{abstract}

KEYWORDS: Deep venous thrombosis; Lipoprotein (a); Meta-analysis; Venous thromboembolism

Lipoprotein (a) [Lp(a)], is a low-density lipoprotein particle linked to apolipoprotein (a). ${ }^{1}$ Over the last decades, numerous studies documented that high plasma $\mathrm{Lp}(\mathrm{a})$ concentrations are associated with a variety of vascular disorders, including peripheral vascular disease, cerebrovascular disease, and coronary heart disease. ${ }^{2-6}$ However, despite the increasing amount of evidence that reports the role of $\mathrm{Lp}(\mathrm{a})$ in the occurrence of arterial thrombosis,

Requests for reprints should be addressed to Francesco Sofi, MD, PhD, Department of Medical and Surgical Critical Care, Thrombosis Centre, University of Florence, Viale Morgagni 85, Florence 50134, Italy.

E-mail address: francescosofi@gmail.com little is known about the role of $\mathrm{Lp}$ (a) on the occurrence of venous thromboembolism. ${ }^{7}$ Some studies investigated the possible association between high Lp(a) plasma levels and venous thromboembolism, but contrasting findings have been obtained. ${ }^{8-12}$ Venous thromboembolism, consisting of deep vein thrombosis and pulmonary embolism, is a potentially fatal disorder and an important cause of morbidity and mortality in industrialized countries. ${ }^{13}$ Most cases of venous thromboembolism arise due to prolonged immobilization, major surgery, trauma, or cancer, but thrombophilic alterations, including elevated $\mathrm{Lp}(\mathrm{a})$ levels, have been reported to have a significant influence on the pathogenesis of the disease. We aimed to conduct a system- 
atic review with meta-analysis of the available studies in order to evaluate the association between elevated $\mathrm{Lp}(\mathrm{a})$ plasma levels and venous thromboembolism.

\section{METHODS}

\section{Search Strategy}

We conducted a computerized search of all publications listed in the electronic databases: MEDLINE (source PubMed, 1966 to November 2006), EMBASE (1980 to November 2006), Web of Science, and The Cochrane Library (source: The Cochrane Central Register of Controlled Trials, 2006, issue 4) using the following text and key words in combination both as MeSH terms and text words: "Lipoprotein (a)," with "venous thrombosis," "deep-vein thrombosis," "venous thromboembolism," "pulmonary embolism." The search strategy had no language restrictions and was supplemented by manually reviewing the reference list of all retrieved articles.

Two investigators (FS, RM) assessed potentially relevant articles for eligibility. The decision to include or exclude studies was hierarchical and initially made on the basis of the study title, then of the study abstract, and finally of the complete study manuscript. In the event of conflicting opinions, resolution of the disagreement was resolved through discussion. Studies were included in the meta-analysis if they had an analytical design (case-control or cohort prospective) and if the patients in the group were aged 18 years or older.

\section{Data Extraction}

Two investigators (FS, RM) independently extracted data using a standardized form. The following baseline characteristics for cases and control groups were collected: number of subjects studied, sex, primary outcome of the study, number of patients, and subjects with $L p(a)$ levels above the considered cut-off value $(>300 \mathrm{mg} / \mathrm{L})$. If the required data could not be located in the published report, we contacted the corresponding author by mail, with a reminder e-mail sent a month later (see Acknowledgements).

\section{Statistical Analysis}

We used Review Manager (RevMan; version 4.2 for Windows; Oxford, UK; The Cochrane Collaboration, 2003) to pool results from the individual studies. Pooled results are reported as odds ratio (OR) and are presented with 95\% confidence interval (CI) with 2 -sided $P$ values using a fixedeffects model (Mantel-Haenszel method), and compared these findings with results obtained using a random-effects model (DerSimonian and Laird method). A $P$ value $<.05$ was considered statistically significant. We considered lev- els of $\mathrm{Lp}$ (a) determining an increased risk for thrombotic event as plasma levels $>300 \mathrm{mg} / \mathrm{L}$, according to the literature. ${ }^{14}$ Statistical heterogeneity was evaluated using the $\mathrm{I}^{2}$ statistic, which assesses the appropriateness of pooling the individual study results. The $\mathrm{I}^{2}$ value provides an estimate of the amount of variance across studies due to the heterogeneity rather than chance. Where $\mathrm{I}^{2}$ was $>50 \%$, heterogeneity was considered substantial. Funnel plots of effect size against standard error were explored, whenever possible, to assess for the presence of publication bias.

\section{RESULTS}

\section{Study Identification and Selection}

The process of our study selection is presented in Figure 1. Our search strategy yielded 24 reports $^{8-12,15-33}$; of them, 12 were excluded on the basis of title/abstract because they studied venous thrombosis in children. ${ }^{15-26}$ Of the remaining 12 articles, one was excluded because it did not report Lp(a) levels but only apoLipoprotein (a) concentrations, ${ }^{27}$ and another study was eliminated because it was, as a matter of fact, a family study that studied the co-segregation of thrombophilic disorders in factor V Leiden carriers. ${ }^{31}$ Among the 10 case-control studies selected to be included in the meta-analysis, however, 3 potentially eligible studies ${ }^{9,32,33}$ were finally excluded because the authors were not able, upon specific request, to collect the exact number of subjects with Lp(a) levels above the con-

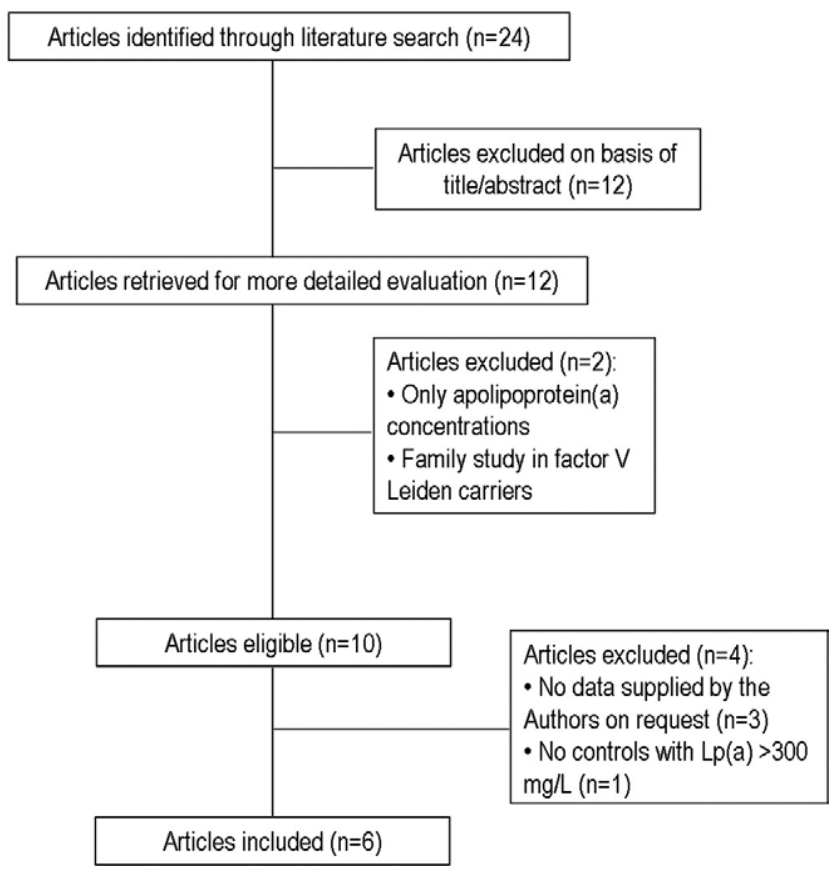

Figure 1 Process of study selection from literature search. 
Table Characteristics of the Studies Included in the Meta-Analysis

\begin{tabular}{|c|c|c|c|c|c|c|c|}
\hline Source & Study Location & $\begin{array}{l}\text { Publication } \\
\text { Year }\end{array}$ & $\begin{array}{l}\text { No. } \\
\text { Cases/Controls }\end{array}$ & Sex & $\begin{array}{l}\text { Index } \\
\text { Event }\end{array}$ & $\begin{array}{l}\mathrm{Lp}(\mathrm{a})>300 \\
\mathrm{mg} / \mathrm{L} \text { in Cases }\end{array}$ & $\begin{array}{l}\mathrm{Lp}(\mathrm{a})>300 \\
\mathrm{mg} / \mathrm{L} \text { in Controls }\end{array}$ \\
\hline Marz et $\mathrm{al}^{8}$ & Frankfurt, Germany & 1990 & $203 / 115$ & $M / F$ & VTE & $56 / 203$ & $30 / 115$ \\
\hline Van Wersch ${ }^{28}$ & Heerlen, The Netherlands & 1994 & $31 / 69$ & $M / F$ & DVT & $1 / 31$ & $7 / 69$ \\
\hline Ignatescu et $\mathrm{al}^{29}$ & Wien, Austria & 1998 & $40 / 50$ & $M / F$ & CTEPH & $24 / 40$ & $0 / 50$ \\
\hline Atsumi et $\mathrm{al}^{30}$ & London, England & 1998 & $31 / 22$ & $M / F$ & DVT in APS & $13 / 31$ & $3 / 22$ \\
\hline von Depka et $\mathrm{al}^{10}$ & Hannover, Germany & 2000 & $685 / 266$ & $M / F$ & VTE & $135 / 685$ & $19 / 266$ \\
\hline Marcucci et al $^{11}$ & Florence, Italy & 2003 & $603 / 430$ & $M / F$ & VTE & $146 / 603$ & $58 / 430$ \\
\hline Vormittag et $\mathrm{al}^{12}$ & Wien, Austria & 2006 & $233 / 122$ & $M / F$ & VTE & $72 / 233$ & $31 / 122$ \\
\hline
\end{tabular}

$\mathrm{VTE}=$ venous thromboembolism; DVT = deep vein thrombosis; CTEPH = chronic thromboembolic pulmonary hypertension; APS = antiphospholipid antibodies syndrome.

sidered cut-off value for an increased risk of thrombotic diseases.

\section{Characteristics of the Studies}

Hence, 7 studies were finally included in the meta-analysis, ${ }^{8-12,28-30}$ for a total number of 1826 cases of venous thromboembolism and 1074 controls. One study was in German. $^{8}$ The study by Ignatescu et $\mathrm{al}^{29}$ reported no controls with $\mathrm{Lp}$ (a) levels $>300 \mathrm{mg} / \mathrm{L}$, so it was excluded from the analysis. Characteristics of the studies included in the meta-analysis are summarized in the Table. Half of the studies reported a significant positive association between $\mathrm{Lp}$ (a) levels $>300 \mathrm{mg} / \mathrm{L}$ and the occurrence of venous thromboembolism. Interestingly, all the studies have been conducted within European countries.

\section{Meta-analysis}

The summary OR under a fixed-effects model showed that patients with $\mathrm{Lp}$ (a) levels above $300 \mathrm{mg} / \mathrm{L}$ had an increased risk of developing venous thromboembolism (OR 1.87; 95\% CI, 1.51-2.30; $P<.0001)$. Although there was significant statistical heterogeneity across the studies $(P=.009$; $\mathrm{I}^{2}=67.4 \%$ ), the random effects model yielded a similar estimate of increased risk (OR 1.77; 95\% CI, 1.14-2.75; $P=.01)$ (Figure 2). Statistical heterogeneity was no longer evident $\left(P=.06 ; \mathrm{I}^{2}=49.3 \%\right)$ when the studies by van Wer$\mathrm{sch}^{28}$ and by Marz et $\mathrm{al},{ }^{8}$ which accounted for the heterogeneity of the model, were excluded from the analysis (OR $2.17 ; 95 \%$ CI, 1.42-3.33; $P=.0004)$. Currently, these 2 studies did not fully report clinical criteria for enrollment of the study patients and one of them included patients with

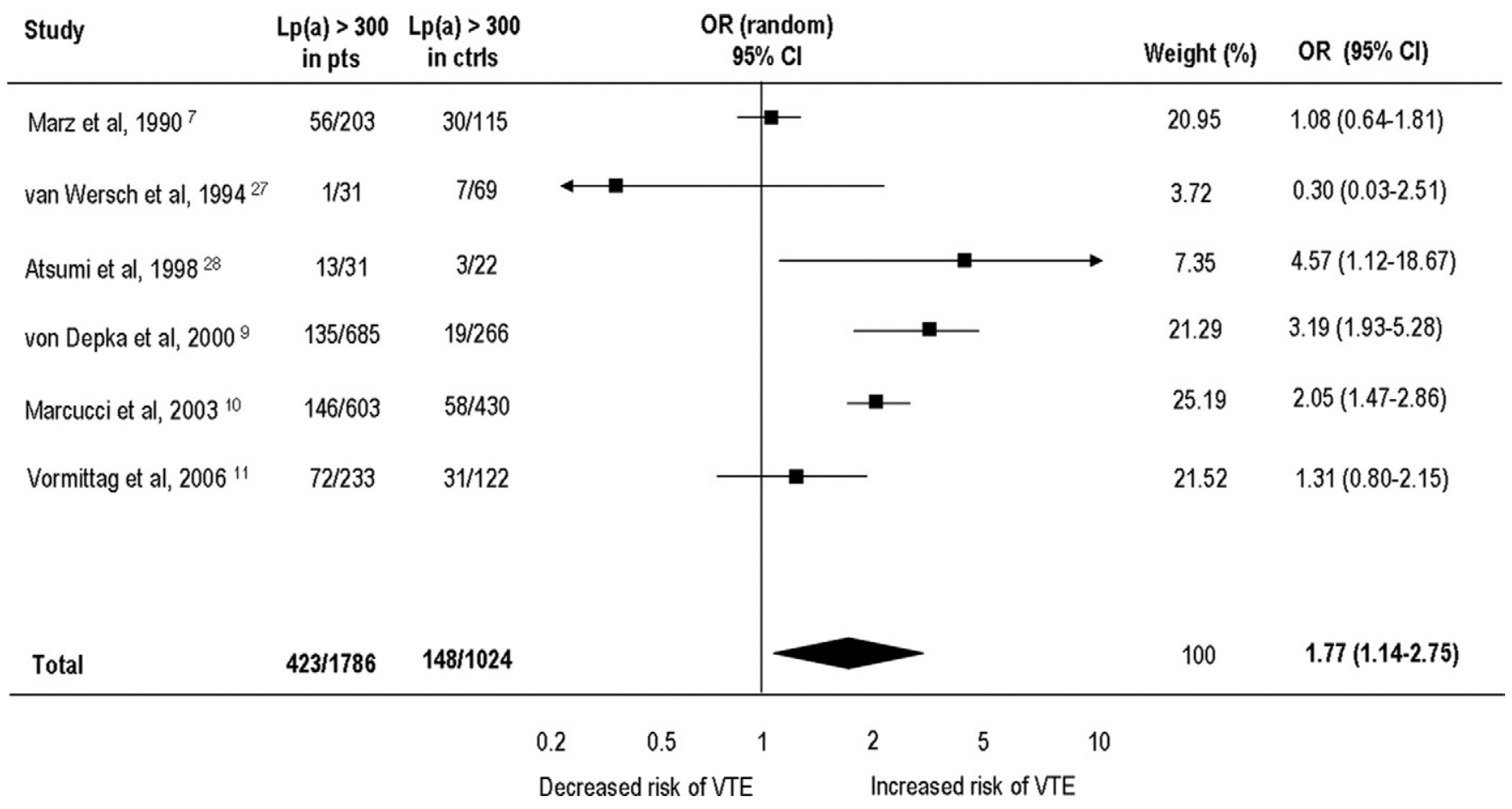

Figure 2 Risk of venous thromboembolism and Lp(a) levels $>300 \mathrm{mg} / \mathrm{L}$. Data are reported as number of subjects with $\mathrm{Lp}(\mathrm{a})>300 \mathrm{mg} / \mathrm{L}$ vs. total number of subjects. 


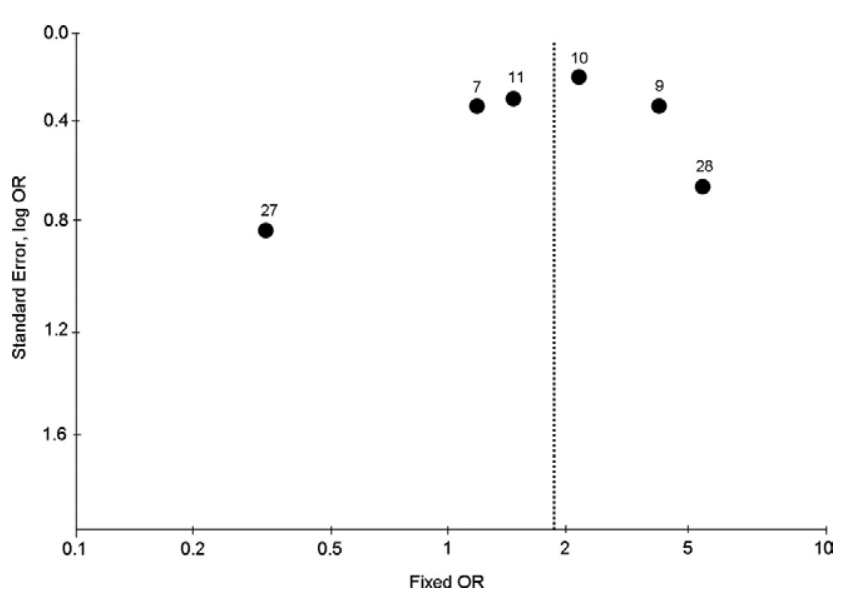

Figure 3 Funnel plot of studies included in the meta-analysis. Number above the dot corresponds to reference number for each study.

various diseases. ${ }^{28}$ The funnel plot of effect size versus standard error performed in order to investigate for a possible publication bias reported to be broadly symmetric, except for the study by Marz et al. ${ }^{27}$ This likely suggests no presence of publication bias among the studies included in the final analysis (Figure 3).

Finally, we performed a sensitivity analysis by taking into consideration only studies that conducted a multivariate analysis, with adjustment for possible confounders, for the association between $\mathrm{Lp}$ (a) $>300 \mathrm{mg} / \mathrm{dL}$ and venous thromboembolism. The summary OR from the 2 studies, ${ }^{10,11}$ which reported data from a multivariate analysis, confirmed the significant association found between high levels of $\mathrm{Lp}$ (a) and an increased risk of venous thromboembolism (OR 2.32; 95\% CI, 1.67-3.24; $P<.0001$ ).

\section{DISCUSSION}

The present meta-analysis performed in 6 case-control studies, with information from an overall incorporated population of nearly 1800 venous thromboembolic patients and 1100 healthy controls shows a significant association between high $\mathrm{Lp}(\mathrm{a})$ levels and the occurrence of venous thromboembolism in adults. Indeed, levels of $\mathrm{Lp}(\mathrm{a})$ above the internationally accepted cut-off of $300 \mathrm{mg} / \mathrm{L}$ determine nearly a 2-fold increased risk of venous thromboembolism. To the best of our knowledge, this is the first systematic review with meta-analysis on studies investigating the association between $L p(a)$ and venous thrombotic disease.

Since its discovery in $1963, \mathrm{Lp}$ (a) has attracted great interest among researchers owing to its particular biochemical structure. Actually, Lp(a) is a specific class of lipoprotein particle composed by a single copy of apolipoprotein B-100 linked to an apo(a) component. ${ }^{1}$ For its similarity with low-density lipoprotein particles, Lp(a) has been thought to have proatherogenic properties. Moreover, Lp(a) also has been demonstrated to have prothrombotic properties, mainly due to the high affinity between the sequence of apo(a) of $\mathrm{Lp}(\mathrm{a})$ and that of the fibrinolytic proenzyme plas- minogen. Currently, the exact mechanism by which $\mathrm{Lp}$ (a) contributes to the process of atherosclerosis or thrombosis is still unclear; however, it seems clear that the role of $\mathrm{Lp}(\mathrm{a})$ in atherogenesis may reflect its involvement in both of these processes, thereby providing a link between the fields of atherosclerosis and thrombosis.

To date, despite the presence of some studies reporting a nonsignificant association between $\mathrm{Lp}(\mathrm{a})$ and vascular diseases, ${ }^{34,35}$ the majority of clinical studies provide evidence for a role of high $\mathrm{Lp}(\mathrm{a})$ levels on the occurrence of atherosclerotic cardiovascular diseases. Indeed, according to international guidelines, $\mathrm{Lp}(\mathrm{a})$ is currently classified as an "emerging" risk factor for cardiovascular diseases. ${ }^{14,36}$ A meta-analysis by Danesh et al, ${ }^{37}$ by evaluating 27 prospective studies and 5436 cases of coronary heart disease observed for a mean follow-up of 10 years, demonstrated that individuals with high $\mathrm{Lp}$ (a) concentrations have an increased risk of coronary heart disease. Moreover, numerous studies have identified elevated $\mathrm{Lp}$ (a) concentrations (mainly $>300 \mathrm{mg} / \mathrm{L}$ ) as a risk factor for a variety of thrombotic vascular disease, including cerebrovascular and peripheral artery disease. ${ }^{3,5}$ More recently, a significant and independent role for $\mathrm{Lp}$ (a) on the progression of atherosclerotic lesion also has been demonstrated. ${ }^{4}$

On the other hand, despite the increasing evidence on the association between $\mathrm{Lp}(\mathrm{a})$ levels and arterial thrombotic diseases, few and contrasting data regarding the role of $\mathrm{Lp}$ (a) levels on the occurrence of venous thromboembolism have been obtained. A positive association with high Lp(a) levels in some $\mathrm{e}^{10,11,29,30}$ but not all the studies has been reported. The contrasting results of the available studies on $\mathrm{Lp}$ (a) and venous thromboembolism can be explained by several issues. Half of the studies available in the literature have been conducted among children, ${ }^{15-26}$ and most of the studies involving adults have investigated less than a hundred cases. ${ }^{28-30}$ In addition, some studies are old and contain scarce and limited information regarding the diagnosis of venous thromboembolism. ${ }^{8,28}$ Moreover, the study populations are not always comparable, including in some cases only women, ${ }^{9}$ patients with antiphospholipid antibodies syndrome,$^{30}$ or patients with other types of diseases. ${ }^{29}$ Accordingly, the differences existing among studies included in the present meta-analysis are clearly reflected by the significant heterogeneity reported in the final analysis. However, the heterogeneity test, after exclusion of the study by Marz et $\mathrm{al}^{8}$ and by van Wersch et $\mathrm{al}^{28}$ that represent the 2 oldest studies containing fewer information on the methodological aspects, lost its statistical significance. Thus, as a final result, the meta-analysis demonstrated a significant association between high levels of $\operatorname{Lp}(\mathrm{a})$ and an increased risk of thrombotic disease affecting the venous district.

However, several potential limitations should be considered, especially because pooling data for meta-analysis purposes from case-control studies implies a relevant problem due to the nonstandardized definition of cases. For example, some of the studies included did not de- 
scribe accurately the methods of diagnosis for venous thromboembolism. ${ }^{7,27}$ Thus, the possibility of false-positive cases cannot be excluded. Another limitation can lay on the possible presence of publication bias: although formal testing did not show any publication bias, such a bias cannot be completely ruled out considering the small number of included studies and the low power of any test to detect publication bias. Furthermore, another bias could be the identification of a threshold level for determining the increased risk of venous thromboembolism. We used as a cut-off value $\mathrm{Lp}$ (a) circulating levels above $300 \mathrm{mg} / \mathrm{L}$. This cut-off has been recently debated by a large prospective study. ${ }^{6}$ However, all the guidelines from the main scientific associations recommend to use this cut-off value for evaluating an increased risk of thrombotic diseases. ${ }^{14,34}$

More detailed combined analysis, perhaps with the use of individual participant data from each study, could help to characterize the real nature of the dose-response relationship, reduce any bias related to the selection of particular cut-off levels, and allow more complete adjustment for other risk factors.

In conclusion, our data point out a special role of $L p(a)$ in venous thromboembolism. This finding could be of relevance especially in the light of the relatively high number of patients with venous thromboembolism and no thrombophilic alterations (among those commonly searched). By adding $\mathrm{Lp}(\mathrm{a})$ assay to the thrombophilic pattern, the number of these "otherwise unexplained" venous thromboembolisms would probably decrease.

\section{ACKNOWLEDGMENTS}

We thank the following authors for kindly providing us additional data: Kurt Huber, MD, University of Wien, Austria, and Jan van Wersch, MD, University of Maastricht, The Netherlands.

\section{References}

1. Berglund L, Ramakrishan R. Lipoprotein (a). An elusive cardiovascular risk factor. Arterioscler Thromb Vasc Biol. 2004;24:2219-2226.

2. Rhoads GG, Dahlen G, Berg K, et al. Lp(a) lipoprotein as a risk factor for myocardial infarction. JAMA. 1986;256:2540-2544.

3. Ariyo AA, Thach C, Tracy R, Cardiovascular Health Study Investigators. Lp(a), lipoprotein, vascular disease, and mortality in the elderly. N Engl J Med. 2003;349:2108-2115.

4. Hartmann M, von Birgelen C, Mintz GS, et al. Relation between lipoprotein (a) and fibrinogen and serial intravascular ultrasound plaque progression in left main coronary arteries. J Am Coll Cardiol. 2006;48:446-452.

5. Ohira T, Schreiner PJ, Morrisett JD, et al. Lipoprotein (a) and incident ischemic stroke. The Atherosclerosis Risk in Communities (ARIC) Study. Stroke. 2006;37:1407-1412.

6. Suk Danik J, Rifai N, Buring JE, Ridker PM. Lipoprotein (a), measured with an assay independent of apoLipoprotein (a) isoform size, and risk of future cardiovascular events among initially healthy women. JAMA. 2006;296:1363-1370.

7. Crowther M. Lipoprotein (a): another risk factor for venous thrombosis? Am J Med. 2003;115:667-668.
8. Marz W, Aygoren E, Trommlitz M, et al. Lipoprotein (a): an indicator of risk in thromboembolic diseases? Klin Wochenschr. 1990;68(Suppl 22):111-112.

9. McColl MD, Sattar N, Ellison J, et al. Lipoprotein (a), cholesterol and triglycerides in women with venous thromboembolism. Blood Coagul Fibrinolysis. 2000;11:225-229.

10. von Depka M, Nowak-Gottl U, Eisert R, et al. Increased lipoprotein (a) levels as an independent risk factor for venous thromboembolism. Blood. 2000;96:3364-3368.

11. Marcucci R, Liotta AA, Cellai AP, et al. Increased plasma levels of lipoprotein (a) and the risk of idiopathic and recurrent venous thromboembolism. Am J Med. 2003;115:601-605.

12. Vormittag R, Vukovich T, Stain M, et al. Lipoprotein (a) in patients with spontaneous venous thromboembolism. Thromb Res. 2006; doi: 10.1016/j.thromres.2006.03.002.

13. Hansson PO, Werlin L, Tibblin G, Eriksson H. Deep vein thrombosis and pulmonary embolism in the general population. Arch Intern Med. 1997; 157:1665-1670.

14. National Cholesterol Education Program (NCEP) Expert Panel on Detection, Evaluation and Treatment of High Blood Cholesterol in Adults (Adult Treatment in Panel III). Third report of the National Cholesterol Education Program (NCEP) expert panel on detection, evaluation, and treatment of high blood cholesterol in adults (Adult Treatment Panel III) final report. Circulation. 2002;106:3143-3421.

15. Munchow N, Kosch A, Schobess R, et al. Role of genetic prothrombotic risk factors in childhood caval vein thrombosis. Eur J Pediatr. 1999;158:109-112.

16. Heller C, Schobess R, Kurnik K, et al. Abdominal venous thrombosis in neonates and infants: role of prothrombotic risk factors-a multicentre case-control study. Br J Haematol. 2000;111:534-539.

17. Kosch A, Junker R, Kurnik K, et al. Prothrombotic risk factors in children with spontaneous venous thrombosis and their asymptomatic parents: a family study. Thromb Res. 2000;99:531-537.

18. Gozdasoglu S, Uysal Z, Ertem M, Akar N. Three risk factors-high lipoprotein (a), elevated FVIII, and FV Leiden-in a pediatric Behcet's disease patient with deep vein thrombosis. Thromb Res. 2002; 106:263.

19. Kosch A, Junker R, Wermes C, Nowak-Gottl U. Recurrent pulmonary embolism in a 13-year-old male homozygous for the prothrombin G20210A mutation combined with protein S deficiency and increased lipoprotein (a). Thromb Res. 2002;105:49-53.

20. Kosch A, Kuwertz-Nroking E, Heller C, et al. Renal venous thrombosis in neonates: prothrombotic risk factors and long-term follow-up. Blood. 2004;104:1356-1360.

21. Kort W, Greiner J, Feldges A, Riesen WF. Increased Lipoprotein (a) levels are not a steady prothrombotic defect. Blood. 2001;98:1993-1994.

22. Nowak-Gottl U, Debus O, Findeisen M, et al. Lipoprotein (a): its role in childhood thromboembolism. Pediatrics. 1997;99:11-13.

23. Nowak-Gottl U, Junker R, Hartmeier M, et al. Increased lipoprotein (a) is an important risk factor for venous thromboembolism in childhood. Circulation. 1999;100:743-748.

24. Nowak-Gottl U, Wermess C, Junker R, et al. Prospective evaluation of the thrombotic risk in children with acute lymphoblastic leukemia carrying the MTHFR TT 677 genotype, the prothrombin G20210A variant, and further prothrombotic risk factors. Blood. 1999;93:1595-1599.

25. Nowak-Gottl U, Junker R, Kreuz W, et al, for the Childhood Thrombophilia Study Group. Risk of recurrent venous thrombosis in children with combined prothrombotic risk factors. Blood. 2001;97:858-862.

26. Revel-Vilk S, Chan A, Bauman M, Massicotte P. Prothrombotic conditions in an unselected cohort of children with venous thromboembolic disease. J Thromb Haemost. 2003;1:915-921.

27. Marz W, Trommlitz M, Scharrer I, Gross W. Apolipoprotein (a) concentrations are not related to the risk of venous thrombosis. Blood Coagul Fibrinolysis. 1991;2:595-599.

28. Van Wersch JWJ. The behaviour of lipoprotein (a) in patients with various diseases. Scand J Clin Lab Invest. 1994;54:559-562. 
29. Ignatescu M, Kostner K, Zorn G, et al. Plasma Lp(a) levels are increased in patients with chronic thromboembolic pulmonary hypertension. Thromb Haemost. 1998;80:231-232.

30. Atsumi T, Khamashta MA, Andujar C, et al. Elevated plasma lipoprotein (a) level and its association with impaired fibrinolysis in patients with antiphospholipid syndrome. J Rheumatol. 1998;25:69-73.

31. Libourel EJ, Bank I, Meinardi JR, et al. Co-segregation of thrombophilic disorders in factor V Leiden carriers; the contributions of factor VIII, factor XI, thrombin activatable fibrinolysis inhibitor and lipoprotein (a) to the absolute risk of venous thromboembolism. Haematologica. 2002;87:1068-1073.

32. Csaszar A, Karadi I, Juhasz E, Romics L. High lipoprotein (a) levels with predominance of high molecular weight apo(a) isoforms in patients with pulmonary embolism. Eur J Clin Invest. 1995;25:368-370.

33. Lippi G, Bassi A, Brocco G, et al. Lipoprotein (a) concentration is not associated with venous thromboembolism in a case control study.
Haematologica. 1999;84:726-729.

34. Moliterno DJ, Jokinen EV, Miserez AR, et al. No association between plasma Lipoprotein (a) concentrations and the presence or absence of coronary atherosclerosis in African-Americans. Arterioscler Thromb Vasc Biol. 1995;15:850-855.

35. Alfthan G, Pekkanen J, Jauhiainen M, et al. Relation of serum homocysteine and Lipoprotein (a) concentrations to atherosclerotic disease in a prospective Finnish population based study. Atherosclerosis. 1994;106:9-19.

36. Marcovina SM, Koschinsky ML, Albers JJ, Skarlatos S. Report of the National Heart, Lung, and Blood Institute workshop on lipoprotein (a) and cardiovascular disease: recent advances and future directions. Clin Chem. 2003;49:1785-1796.

37. Danesh J, Collins R, Peto R. Lipoprotein (a) and coronary heart disease. Meta-analysis of prospective studies. Circulation. 2002;102: 1082-1085. 